\title{
Isoprostanes and Oxidative Stress in Off-Pump and On-Pump Coronary Bypass Surgery
}

\author{
Viviana Cavalca, Biol Sci, Erminio Sisillo, MD, Fabrizio Veglia, PhD, Elena Tremoli, PhD, \\ Giuliana Cighetti, PhD, Luca Salvi, MD, Alessandra Sola, PhD, Luciana Mussoni, PhD, \\ Paolo Biglioli, MD, Giancarlo Folco, PhD, Angelo Sala, PhD, and
} Alessandro Parolari, MD, PhD

Centro Cardiologico Monzino IRCCS, Institute of Cardiology, Department of Pharmacological Sciences, Department of Medical Chemistry, Biochemistry, and Biotechnology, and Center for Cardiopulmonary Pharmacology, University of Milan, Milan, Italy

Background. Conventional on-pump coronary artery bypass grafting (CABG) is associated with a systemic inflammatory response and by an increased production of reactive oxygen species, whereas off-pump coronary artery bypass grafting (OPCAB) is thought to be accompanied by less oxidative stress. Urinary isoprostane $\mathrm{iPF}_{2 \alpha}-\mathrm{III}$ is a new marker reflecting oxidative stress; it has emerged as the most reliable marker of oxidative stress status in vivo. This study was designed to ascertain whether OPCAB compared with CABG represents a surgical strategy that avoids oxidative stress. To this end urinary isoprostanes and other established oxidative stress markers were measured during the first 24 hours after CABG and OPCAB.

Methods. Fifty low-risk coronary patients were randomly assigned to CABG or OPCAB. Urinary isoprostane $\mathrm{iPF}_{2 \alpha}$-III levels, plasma levels of free malondialdehyde, and total antioxidant status were measured before, during, and up to 24 hours after surgery.

$\mathrm{C}$ onventional on-pump coronary artery bypass grafting (CABG) has been previously shown [1-5] to be associated with prothrombotic and proinflammatory response. During this type of surgical intervention reactive oxygen species (ROS) are also produced, together with neutrophil sequestration and activation within vasculature, endothelial damage, and increased vascular permeability. An increase in ROS can overwhelm local antioxidant defense mechanisms and cause damage to biological molecules; eg, DNA, lipids, and proteins. Altogether these effects may represent important contributory factors to the incidence of perioperative or postoperative complications occurring after CABG [6].

The avoidance of cardiopulmonary bypass (CPB), as well as of heart and lung ischemia-reperfusion, has been proposed to reduce the postoperative systemic inflammatory response in off-pump coronary artery bypass grafting (OPCAB). In fact, OPCAB is associated with a somehow lower degree of systemic inflammatory and oxidative response than conventional $\mathrm{CABG}$ during the

Accepted for publication Aug 18, 2005.

Address correspondence to Dr Parolari, Centro Cardiologico Monzino IRCCS, Via Carlo Parea 4, 20138 Milan, Italy; e-mail: aparolari@ccfm.it.
Results. In OPCAB $\mathrm{iPF}_{2 \alpha}$-III excretion remained unchanged throughout the study. As expected, in CABG $\mathrm{iPF}_{2 \alpha}$-III levels significantly increased during surgery and returned at baseline 24 hours later. Free malondialdehyde behaved similarly, with no change in ОРСАВ and sharp increases during CABG. Conversely, total antioxidant status showed a sharp drop during CABG, followed by a slow recovery, whereas a significantly lower drop occurred in OРСАВ.

Conclusions. In this randomized study in low-risk coronary patients, OPCAB revealed less perioperative oxidative stress, as reflected by lack of excretion of $\mathrm{iPF}_{2 \alpha}$-III in urine, by lack of increase of plasma free malondialdehyde, and by lower decreases in plasma total antioxidant status.

(Ann Thorac Surg 2006;81:562-7)

(C) 2006 by The Society of Thoracic Surgeons

intraoperative period and the very early hours after surgery $[1,5,7,8]$.

The $\mathrm{F}_{2}$ isoprostanes are a family of free radical catalyzed prostaglandin $F_{2}$ isomers that are formed in situ from the fatty acid backbone esterified in membrane phospholipids. They are released in response to cellular activation and have been detected in human plasma and urine $[9,10]$. Increased excretion of $F_{2}$ isoprostanes in urine has been found in association with advanced age [11], chronic obstructive pulmonary disease [12], cigarette smoking [13], and hypercholesterolemia [14]. Due to their mechanism of formation, specific structural features, and chemical stability, they are considered a reliable index of oxidant stress and ensuing lipid peroxidation in vivo [15]. An increase in the urinary excretion of $F_{2}$ isoprostanes has been previously reported in CABG [16], but we have found no information on isoprostane excretion available in patients undergoing OPCAB.

The present study tests the hypothesis that the increases in oxidative markers occurring during CABG are prevented in patients undergoing OPCAB. To this end we assessed the in vivo secretion of urinary excretion of $\mathrm{F}_{2}$ isoprostanes and the levels in plasma of some established markers of oxidative stress in patients undergoing 
CABG or OPCAB. In addition, the potential correlation of urinary excretion of $F_{2}$ isoprostanes with some markers of oxidative stress in plasma was also evaluated.

\section{Patients and Methods}

Fifty first-time isolated low-risk (EuroSCORE $<6$ ) coronary bypass surgery patients, for whom an on-pump and an off-pump procedure was deemed technically feasible and not contraindicated (ie, porcelain aorta for CABG), were enrolled in accordance with a protocol approved by the Institutional Review Board (approved January 11, 2002) at the Centro Cardiologico Monzino, and written informed consent was obtained. Exclusion criteria were Q-wave myocardial infarction in the last 6 weeks, unstable angina, or poor left ventricular function (ejection fraction $[\mathrm{EF}]<0.30$ ), whereas there were no restrictions for age.

Patients were randomly assigned to CABG $(n=25)$ or OPCAB $(n=25)$. The same surgical and anesthetic team managed all patients. None of the patients were taking vitamins, dietary supplements, or drugs with established antioxidant properties during the study. Conventional therapy was allowed according to clinical judgment.

After internal mammary harvesting, heparin (300 IU/ $\mathrm{kg}$ ) was given and activated clotting time was kept 440 seconds or greater with additional heparin in both groups. Upon completion of distal and proximal coronary anastomoses, heparin was antagonized with protamine sulfate at a 1:1 ratio.

\section{$C A B G$}

A nonpulsatile roller pump and hollow-fiber oxygenators were used. Cardiopulmonary bypass was initiated with cannulas placed in the ascending aorta and right atrium (two-stage venous cannula). Each operation was performed with tepid hypothermia $\left(32-33^{\circ} \mathrm{C}\right)$ and hemodilution. During CPB, blood flow was maintained at 2.4 $\mathrm{L} \cdot \mathrm{min} \cdot \mathrm{m}^{2}$. Myocardial protection was achieved by the administration of cool, antegrade and retrograde multidose blood cardioplegia.

\section{$O P C A B$}

This procedure was performed by a midline sternotomy with the same heparinization protocol as described above. Mechanical stability of the coronary arteriotomy area was achieved with the Octopus IV system (Medtronic Inc, Minneapolis, MN) and a soft plastic coronary flow-shunt was always passed into the coronary arteriotomy to reduce myocardial ischemia and to improve visualization of the anastomosis field. The hemodynamic management of patients during distal coronary anastomosis consisted mainly in the careful and progressive elevation of the heart with tissue slices, associated with substantial volume administration to allow the heart to adapt to the new positioning and to avoid major hemodynamic derangements or need of inotropic drug administration.

\section{Urine Sampling}

An overnight urine collection the night before (pre), 4 to 6 hours after surgery start (during), and an overnight urine collection 24 hours after surgery (after) was carried out. The antioxidant 4-hydroxy-tempo ( $1 \mathrm{mmol} / \mathrm{L}$; Sigma Chemical Co, St Louis, MO) was added to urine and samples stored at $-80^{\circ} \mathrm{C}$ until analyzed.

\section{Blood Sampling}

Blood samples were collected at 7 time-points, from a catheter positioned in the radial artery, in tubes containing ethylenediaminetetraacetic acid $(9.3 \mathrm{mM}$; Vacutainer Systems, Becton Dickinson, Rutherford, NY) as anticoagulant: before induction of anesthesia ( $t 0$, pre), after sternotomy (t1), 30 minutes after aortic cross-clamp in CABG and 30 minutes after the start of the first distal anastomosis in OPCAB $(t 2)$, after protamine administration $(t 3)$, at the end of surgery ( $t 4$, about 1.5 hours from $t 2), 4$ hours after the arrival to the intensive care unit ( $t 5)$, and 24 hours after surgery (t6). After blood centrifugation, plasma was removed and stored at $-80^{\circ} \mathrm{C}$ until analyzed.

\section{Isoprostane Determination}

Urinary isoprostane $\mathrm{PPF}_{2 \alpha}$-III was purified using a double extraction protocol followed by quantification using an enzyme-immunoassay (Cayman Chemical Co, Ann Arbor, MI - SPI-BIO, Saclay, France), according to Wang and colleagues [11], with modification, as previously described [17]. A 20,000 dpm of [ $\left.{ }^{3} \mathrm{H}\right]-\mathrm{PGF}_{2 \alpha}$ was added as internal standard to $2 \mathrm{~mL}$ of urine and results were corrected for the recovery and expressed as picogram per milligram of creatinine. Only free (not esterified in phospholipids) isoprostanes can be detected.

\section{Assay of Free Plasma Malondialdehyde (MDA)}

Free plasma MDA levels were assessed, after organic extraction, with gas chromatography-mass spectrometry (GC-MS) as previously described [18]. Synthesized dideuterated-MDA was added as internal standard. The GC-MS analyses were carried out on a Hewlett-Packard 5890 gas chromatograph (HP Company, Palo Alto, CA) equipped with an HP-5 fuse silica capillary column $(25 \mathrm{~m}$, $0.32 \mathrm{~mm}$ id, $0.25 \mathrm{~mm}$ film thickness) and coupled to a 5988A mass spectrometer.

\section{Assay of Individual Antioxidant Status (IAS)}

Plasma IAS was measured by a commercially available spectrophotometric assay (OXY-Adsorbent Test, Diacron International, Grosseto, Italy). Plasma samples were submitted to massive oxidative stress with hypochlorous acid and IAS values determined by reading the absorbance at $505 \mathrm{~nm}$.

\section{Statistical Analysis}

Normally distributed data are reported as means \pm 1 standard deviation; data that were not normally distributed are reported as median and interquartile range (IQR) between brackets. Clinical variables of the patients were compared with two sample t-tests, $\chi^{2}$, or Fisher's 
Table 1. Main Characteristics of the Patients

\begin{tabular}{lccc}
\hline & $\begin{array}{c}\text { CABG } \\
(\mathrm{n}=25)\end{array}$ & $\begin{array}{c}\text { OPCAB } \\
(\mathrm{n}=22)\end{array}$ & $\begin{array}{c}p \\
\text { Value }\end{array}$ \\
\hline Age & $63.4 \pm 11.5$ & $68.4 \pm 8.9$ & 0.11 \\
Males (\%) & $17(68)$ & $18(82)$ & 0.33 \\
Hypertension (\%) & $16(64)$ & $12(55)$ & 0.72 \\
Diabetes (\%) & $7(28)$ & $5(23)$ & 0.94 \\
Previous acute myocardial & $12(48)$ & $8(36)$ & 0.61 \\
$\quad$ infarction (\%) & & & \\
Chronic obstructive & $1(5)$ & $2(8)$ & $>0.99$ \\
$\quad$ pulmonary disease (\%) & & & \\
Chronic renal failure (\%) & $3(14)$ & $0(0)$ & 0.24 \\
Ejection fraction (\%) & $56.2 \pm 13.4$ & $55.8 \pm 9.6$ & 0.91 \\
Distal anastomoses & $3.1 \pm 0.67$ & $2.5 \pm 0.67$ & 0.005 \\
\hline
\end{tabular}

$\mathrm{CABG}=$ coronary artery bypass grafting; $\quad \mathrm{OPCAB}=$ off-pump coronary artery bypass.

exact tests when indicated. Values represented in time course charts are the mean differences (delta \pm standard error of the mean) between each time point value and that measured at baseline.

In order to adjust for potential confounding factors (ie, age, gender, EF, previous myocardial infarction, and number of bypass performed), general linear model analysis of covariance models were used for statistical analysis of time, group (CABG vs OPCAB), and interaction (time* group) effects in oxidative stress variables; in this case skewed variables $\left(\mathrm{iPF}_{2 \alpha}-\mathrm{III}\right)$ were logtransformed before analysis. When time, group, or interaction effects were significant $(p<0.05)$, repeated measures ANCOVA with Bonferroni correction was used to establish significant $(p<0.05)$ point-by-point differences.

To compute within subjects' correlations we first subtracted individual patient means from all values. The resulting residuals were then analyzed by Spearman correlation. All analyses were performed by SAS statistical package v.8 (SAS Institute Inc, Cary, NC).

\section{Results}

\section{Clinical Characteristics and Surgical Data}

Forty-seven out of the 50 randomized patients successfully completed the study. Three, all in the OPCAB group were excluded for the following reasons: refusal to undergo surgery after randomization (1 patient), conversion to CABG (1 patient), and perioperative myocardial infarction (1 patient). There were no significant differences between groups both in preoperative and intraoperative variables, except for the mean number of grafts implanted (Table 1); all patients who completed the protocol had a normal postoperative course.

\section{Urine Isoprostane Excretion, Plasma MDA, and Total Antioxidant Status}

Before surgical intervention, isoprostane excretion of $\mathrm{iPF}_{2 \alpha}$-III in urine was $98.5(67.5-155.5 \mathrm{IQR})$ and 99.5 (56-128 IQR) $\mathrm{pmol} / \mathrm{mmol}$ creatinine in patients undergoing $\mathrm{OPCAB}$ and $\mathrm{CABG}$, respectively (Table 2 ). In patients undergoing OPCAB urinary $\mathrm{iPF}_{2 \alpha}$-III, excretion did not change significantly during the study; on the other hand, a consistent increase in isoprostane excretion was detected in CABG patients during surgery (153 pmol/mmol creatinine, 112-257 IQR, $p<0.01$ vs presurgery), levels returning to baseline 24 hours after surgery (Fig 1A, and Table 2). Similarly, no change in plasma free MDA levels were observed in OPCAB patients, whereas in CABG patients free MDA was significantly higher than baseline and than OPCAB patients from the time point 30 minutes after aortic cross-clamp application (t2) up to surgery end (t4), and peaking after protamine administration (t3) (Fig $1 \mathrm{~B}$, and Table 2). Total MDA levels in plasma behaved as free MDA (data not shown).

Interestingly, a statistically significant reduction of IAS occurred both in CABG and OPCAB starting from the time point 30 minutes after aortic cross-clamp application (t2) and lasting up to 24 hours after surgery, the decrease being more marked in CABG at all time points. The IAS levels tended to return to baseline 24 hours after surgery but the recovery was incomplete both in CABG and in OPCAB (Fig 1C and Table 2). After adjustment for age, gender, EF, previous myocardial infarction, and number of bypass performed, data remained substantially unchanged for the three variables $\left(\mathrm{iPF}_{2 \alpha}-\mathrm{III}\right.$, free MDA, IAS) considered.

In CABG patients a positive $(\mathrm{r}=0.48, p<0.0001)$ correlation was found between the changes in urinary $\mathrm{iPF}_{2 \alpha}$-III and plasma free MDA. In addition, changes in $\mathrm{iPF}_{2 \alpha}$-III and in plasma free MDA negatively correlated with changes in IAS $(r=-0.53, p<0.0001$ and $r=-0.46$, $p<0.0001$, respectively).

\section{Comment}

This study shows that off-pump coronary surgery markedly reduces the occurrence of an in vivo prooxidant state; the lack of changes in the levels of $\mathrm{iPF}_{2 \alpha}-\mathrm{III}$ in urine and of free MDA in plasma, together with an only modest reduction of plasma total antioxidant status in OPCAB, suggests that the contribution of general surgical trauma to the oxidative stress is minimal and affects only the endogenous antioxidant defense mechanism without sensibly increasing the production of prooxidant species.

Altered levels of immunoreactive $\mathrm{iPF}_{2 \alpha}-\mathrm{III}$ in urine were reported in the clinical setting of coronary reperfusion [15] and in a variety of syndromes putatively associated with oxidant stress in vivo [16]. Moreover, isoprostanes are known to have biological effects in vitro through membrane receptors for prostanoids $[19,20]$ and may therefore be considered themselves as mediators of oxidative stress.

Our study does not answer the question of which cells or tissues, subjected to oxidant stress, contribute to the increased isoprostanes excretion and MDA formation detected during surgery in CABG patients. One likely source might be blood cells stressed when circulating through the heart-lung machine. Indeed, activation of granulocytes and subsequent release of oxygen-derived free radicals and granular enzymes are well-recognized 
Table 2. Measured Oxidative Stress Markers Over Time

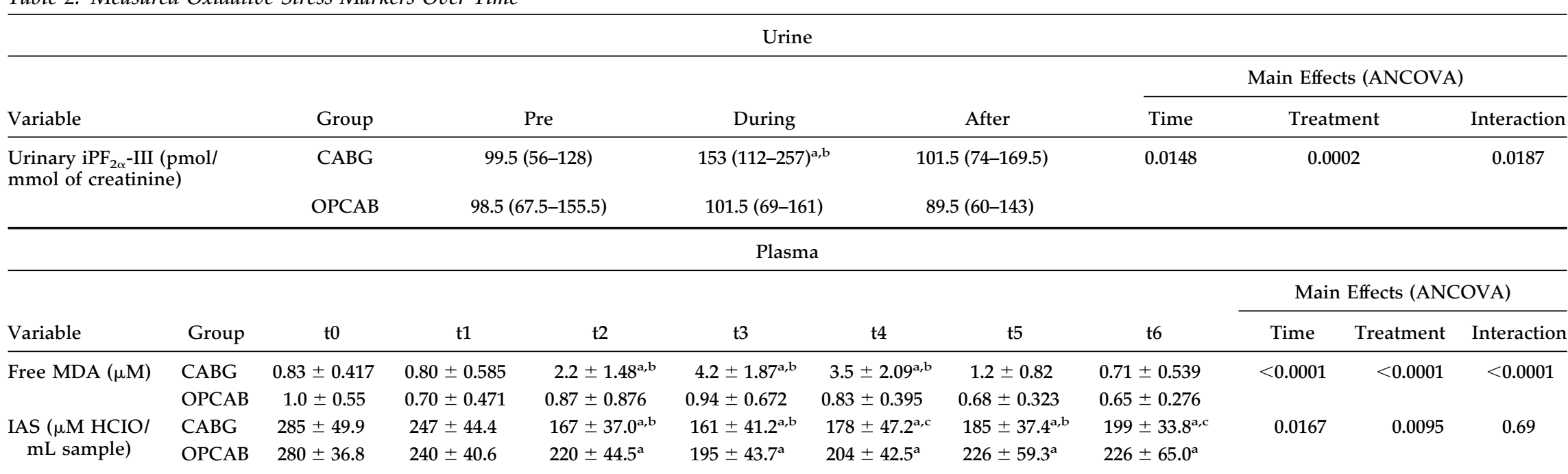

$\mathrm{a}=$ Significant difference $(p<0.01$, repeated measures ANCOVA) within each group as compared with baseline; $\quad \mathrm{b}=$ Significant difference $(p<0.01$, repeated measures ANCOVA) between CABG and OPCAB groups; $\quad{ }^{c}=$ Significant difference $(p<0.05$, repeated measures ANCOVA) between CABG and OPCAB groups.

ANCOVA $=$ analysis of covariance; $\quad$ CABG $=$ coronary artery bypass grafting; $\quad$ HCIO $=$ hypochlorous acid $; \quad$ IAS $=$ individual antioxidant status; $\quad$ MDA $=$ malondialdehyde 
A

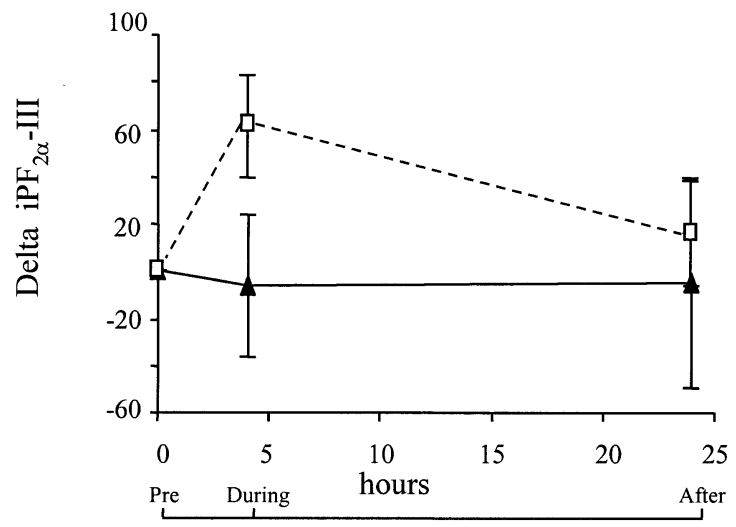

B

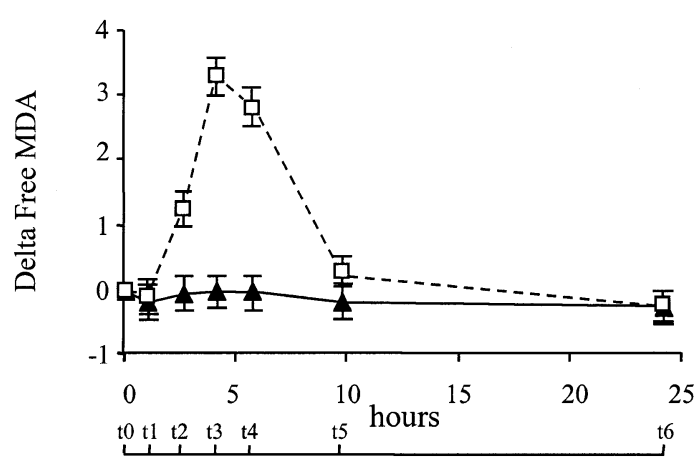

C

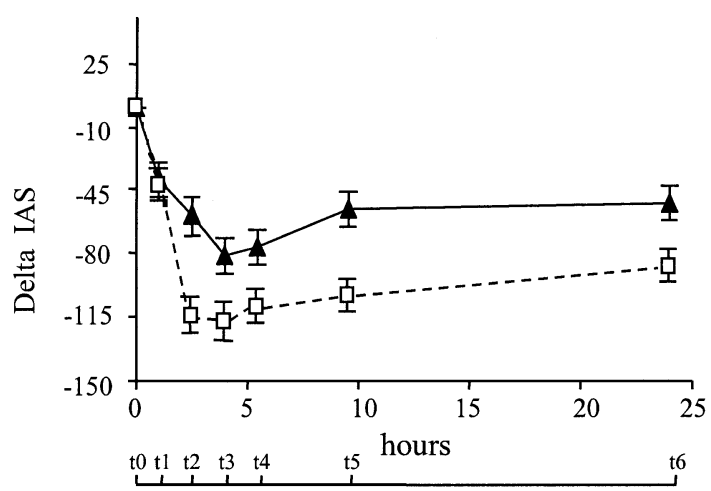

Fig 1. Perioperative difference (delta) in urinary $i P F_{2 \alpha}-I I I$ excretion (A), plasma free malondialdehyde (MDA) levels (B), and plasma total individual antioxidant status (IAS) (C). Values represented in time course charts are the differences (delta \pm standard error of the mean) between each time point value and that measured at baseline. $(\square=$ coronary artery bypass grafting; $\boldsymbol{\Delta}=$ off-pump coronary artery bypass.)

during cardiac surgery with the use of CPB. Possible sites of leukocyte activation might be the coagulation and fibrinolytic cascades as well as complement activation, likely through the alternative pathway (eg, through blood-circuit contact) [3, 21]. Activated platelets may represent a direct source of isoprostanes as these cells might be significantly triggered by contact activation with the material surface of the heart-lung machine and are able to synthesize isoprostanes in a cyclooxygenasedependent fashion [22]. Nonetheless, activated platelets may generate isoprostanes and thromboxane B2 in a molar ratio of approximately 1:1000 and it therefore seems difficult to link the increased concentrations of urinary $\mathrm{iPF}_{2 \alpha}$-III simply with CPB-related platelet activation.

The sharp drop in IAS status detected in CABG patients may reflect an augmented utilization of plasma antioxidants, whereas the increases in $\mathrm{iPF}_{2 \alpha}-\mathrm{III}$ and MDA suggest an enhanced oxidative stress. Thus, in CABG patients, free radical generation outweighs the endogenous defense mechanisms and may contribute to global myocardial reperfusion injury [23-25], whereas in OPCAB patients only a reduction in endogenous defense mechanisms was observed, as reflected by a relatively modest, but significant, reduction of IAS.

Thus, OPCAB minimizes intraoperative oxidative stress. Whether or not this protection is enough to prevent short-term or long-term clinical events is not addressed in this trial and requires further investigation. Up to the present time, multiple clinical studies have examined the issue whether or not off-pump coronary grafting is associated with lower mortality and morbidity rates without gaining a consensus [26-28]. In fact, no evidence coming from large, randomized controlled trials is available on these issues and all the available information comes mostly from observational, uncontrolled, nonrandomized clinical trials, which makes their generalization difficult [29].

In conclusion, our results indicate that patients undergoing OPCAB had only mild signs of oxidative stress compared with patients submitted to CABG. Further studies are needed to investigate whether this improved protection against oxidation will result in improved clinical outcomes.

\section{References}

1. Matata BM, Sosnowski AW, Galinanes M. Off-pump bypass graft operation significantly reduces oxidative stress and inflammation. Ann Thorac Surg 2000;69:785-91.

2. Wan S, DeSmet JM, Barvais L, Goldstein M, Vincent JL, LeClerc JL. Myocardium is a major source of proinflammatory cytokines in patients undergoing cardiopulmonary bypass. J Thorac Cardiovasc Surg 1996;112:806-11.

3. Fransen E, Maessen J, Dentener M, Senden N, Geskes G, Buurman W. Systemic inflammation present in patients undergoing $C A B G$ without extracorporeal circulation. Chest 1998;113:1290-5.

4. Dybdahl B, Wahba A, Lien E, et al. Inflammatory response after open heart surgery: release of heat-shock protein 70 and signaling through toll-like receptor-4. Circulation 2002; 105:685-90.

5. Schulze C, Conrad N, Schutz A, et al. Reduced expression of systemic proinflammatory cytokines after off-pump versus conventional coronary artery bypass grafting. Thorac Cardiovasc Surg 2000;48:364-9.

6. Edmunds LH. Inflammatory response to cardiopulmonary bypass. Ann Thorac Surg 1998;66:S12-6.

7. Biglioli P, Cannata A, Alamanni F, et al. Biological effects of off-pump vs. on-pump coronary artery surgery: focus on inflammation, hemostasis and oxidative stress. Eur J Cardiothorac Surg 2003;24:260-9. 
8. Gerritsen WB, van Boven WJ, Driessen AH, Haas FJ, Aarts LP. Off-pump versus on-pump coronary artery bypass grafting: oxidative stress and renal function. Eur J Cardiothorac Surg 2001;20:923-9.

9. Morrow JD, Minton TA, Mukundan CR, et al. Free radicalinduced generation of isoprostanes in vivo. Evidence for the formation of D-ring and E-ring isoprostanes. J Biol Chem 1994;269:4317-26.

10. Patrono C, FitzGerald GA. Isoprostanes: potential markers of oxidant stress in atherothrombotic disease. Arterioscler Thromb Vasc Biol 1997;17:2309-15.

11. Wang Z, Ciabattoni G, Creminon C, et al. Immunological characterization of urinary 8-epi-prostaglandin F2 alpha excretion in man. J Pharmacol Exp Ther 1995;275:94-100.

12. Pratico D, Basili S, Vieri M, Cordova C, Violi F, Fitzgerald GA. Chronic obstructive pulmonary disease is associated with an increase in urinary levels of isoprostane F2alpha-III, an index of oxidant stress. Am J Respir Crit Care Med 1998;158:1709-14.

13. Reilly M, Delanty N, Lawson JA, FitzGerald GA. Modulation of oxidant stress in vivo in chronic cigarette smokers. Circulation 1996;94:19-25.

14. Davi G, Alessandrini P, Mezzetti A, et al. In vivo formation of 8-epi-prostaglandin F2 alpha is increased in hypercholesterolemia. Arterioscler Thromb Vasc Biol 1997;17:3230-5.

15. Delanty N, Reilly MP, Pratico D, et al. 8-epi $\mathrm{PGF}_{2 \alpha}$ generation during coronary reperfusion: a potential quantitative marker of oxidant stress in vivo. Circulation 1997;95:2492-9.

16. Lawson JA, Rokach J, FitzGerald GA. Isoprostanes: formation, analysis and use as indices of lipid peroxidation in vivo. J Biol Chem 1999;274:24441-4.

17. Visioli F, Galli C, Plasmati E, et al. Olive phenol hydroxytyrosol prevents passive smoking-induced oxidative stress. Biochem Biophys Res Commun 2000;30:797-9.

18. Cighetti G, Debiasi S, Paroni R, Allevi P. Free and total malondialdehyde assessment in biological matrices by gas chromatography-mass spectrometry: what is needed for an accurate detection. Anal Biochem 1999;266:222-9.
19. Yin K, Halushka PV, Yan YT, Wong PY. Antiaggregatory activity of 8-epi-prostaglandin F2 alpha and other F-series prostanoids and their binding to thromboxane A2/ prostaglandin $\mathrm{H} 2$ receptors in human platelets. J Pharmacol Exp Ther 1994;270:1192-6.

20. Wagner RS, Weare C, Jin N, Mohler ER, Rhoades RA. Characterization of signal transduction events stimulated by 8-epi-prostaglandin(PG)F2 alpha in rat aortic rings. Prostaglandins 1997;54:581-99.

21. Beckman JS, Beckman TW, Chen J, Marshall PA, Freeman BA. Apparent hydroxyl radical production by peroxynitrite: implications for endothelial injury from nitric oxide and superoxide. Proc Natl Acad Sci U S A 1990;87:1620-4.

22. Pratico D, Lawson JA, FitzGerald GA. Cyclooxygenasedependent formation of the isoprostane, 8-epi prostaglandin F2 alpha. J Biol Chem 1995;270:9800-8.

23. Loesser KE, Kukreja RC, Kazziha SY, Jesse RL, Hess ML. Oxidative damage to the myocardium: a fundamental mechanism of myocardial injury. Cardioscience 1991;2:199-216.

24. Bolli R. Myocardial 'stunning' in man. Circulation 1992;86: 1671-91.

25. Ferreira R, Llesuy S, Milei J, et al. Assessment of myocardial oxidative stress in patients after myocardial revascularization. Am Heart J 1988;115:307-12.

26. Légaré JF, Buth KJ, King S, et al. Coronary bypass surgery performed off-pump does not result in lower in-hospital morbidity than coronary artery bypass grafting performed on-pump. Circulation 2004;109:887-92.

27. Lytle BW, Sabik JF. On-pump and off-pump bypass surgery: tools for revascularization. Circulation 2004;109:810-2.

28. Parolari A, Biglioli P, Alamanni F. Improved early outcomes after OPCAB: when will the final answer come? Circulation 2004;13;109:e181.

29. Parolari A, Alamanni F, Cannata A, et al. Off-pump versus on-pump coronary artery bypass: meta-analysis of currently available randomized trials. Ann Thorac Surg 2003;76:37-40. 